\title{
Avaliação da qualidade tecnológica de snacks obtidos por extrusão de grão integral de amaranto ou de farinha de amaranto desengordurada e suas misturas com fubá de milho
}

Quality assessment of snacks obtained by extrusion of whole amaranth grains or defatted amaranth flour and their mixtures with corn grits

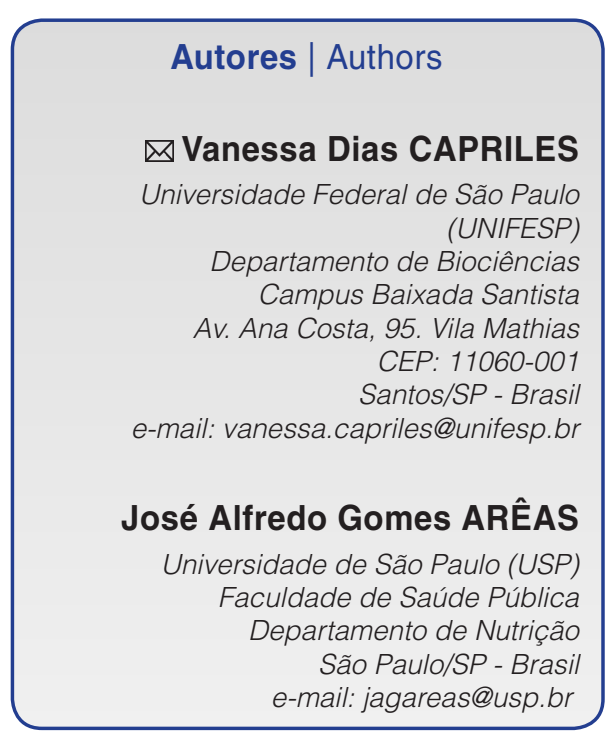

$\triangle$ Autor Correspondente / Corresponding Author

Recebido / Received: 27/05/2010 Aprovado / Approved: 20/09/2011 Publicado / Published: mar./2012

\section{Resumo}

Apesar dos benefícios nutricionais e de seu potencial como alimento funcional, o amaranto ainda é um alimento pouco difundido no Brasil. Para o aumento do consumo desta matéria-prima, preconiza-se a sua incorporação na formulação de produtos convencionais, como os snacks extrudados que vêm sendo elaborados com a farinha de amaranto desengordurada. Pouco tem sido pesquisado sobre a extrusão direta do grão integral de amaranto, o que permitiria eliminar as etapas de moagem e de desengorduramento. Assim, a pesquisa avaliou e comparou a qualidade tecnológica de snacks obtidos por extrusão de grão integral de amaranto e de farinha de amaranto desengordurada, e suas misturas com 25 e $50 \%$ de fubá de milho. Verificou-se que o teor de lipídeos presentes no grão de amaranto (8\%) prejudicou a expansão e a textura dos extrudados. Mesmo com a adição de fubá de milho, os snacks extrudados à base de grão integral de amaranto foram rejeitados sensorialmente (aceitação global entre 3 e 4 , numa escala hedônica estruturada de nove pontos), pois apresentaram baixa expansão, textura dura, cor escura e forte sabor residual. Já os snacks extrudados obtidos com farinha de amaranto desengordurada isoladamente e em combinação com fubá de milho apresentaram maior expansão, textura crocante e cor mais clara, e, por isso, maior aceitabilidade (aceitação global $\geq 5$ ). Conclui-se, nas condições do experimento, que não foi possível obter snacks extrudados à base de grãos de amaranto de boa aceitação e que a produção de snacks extrudados à base de farinha de amaranto desengordurada proporciona produtos com melhor aceitação por parte do consumidor.

Palavras-chave: Amaranthus cruentus; Extrudados expandidos diretos; Aceitabilidade.

\section{Summary}

Despite its nutritional benefits and potential as a functional food, amaranth is still little known in Brazil. In order to increase its consumption, the inclusion of amaranth in the formulation of conventional products has been recommended, such as in extruded snacks, which are being prepared with defatted amaranth flour. There has been little research on the direct extrusion of whole amaranth grains, which would eliminate the grinding and defatting processes. Thus, this study evaluated and compared the quality of snacks obtained by the extrusion of whole amaranth grains and defatted amaranth flour and their mixtures with 25 and $50 \%$ corn grits. The lipid content present in the amaranth grain (8\%) impaired the expansion and texture of the extrudates. Even with the addition of corn, the snacks based on whole amaranth grains were not well accepted (overall acceptability between 3 and 4 on a nine point hedonic scale), due to their low expansion, hard texture, dark color and strong aftertaste. On the other hand, snacks made with defatted amaranth flour and its mixtures with corn grits showed good expansion and had a crunchy texture and lighter color, and were therefore better accepted (overall acceptability $\geq 5$ ). It was concluded that under the present experimental conditions, it was not possible to develop an acceptable snack based on whole amaranth grains, but the production of snacks based on defatted amaranth flour provided products better accepted by the consumers. 


\section{Introdução}

O amaranto é provavelmente originário das Américas do Sul e Central. Representou a base da dieta de diversas civilizações pré-colombianas, dentre as quais os Maias, Incas e Astecas. No período colonial, em consequência da pressão exercida pelos espanhóis, o consumo e o cultivo do amaranto foram proibidos. Com isso, a cultura quase desapareceu, mantendo-se apenas em algumas regiões andinas de forma incipiente. Em 1975, o amaranto ressurge mundialmente, quando a Academia Nacional de Ciências dos Estados Unidos da América o considera como uma das culturas mais promissoras para alimentar a humanidade em função do seu valor nutritivo (TEUTONICO e KNORR, 1985). Desde então, houve um significativo aumento na atividade de pesquisa envolvendo o amaranto. A literatura vem apontando os benefícios nutricionais e funcionais desse alimento, e, atualmente, há áreas de cultivo distribuídas em todos os continentes, mas ainda não existem dados oficiais de produção mundial (FERREIRA et al., 2007).

A planta apresenta capacidade de adaptação a diferentes condições de solo e clima, podendo ser produzida no nível do mar e até em altitudes superiores a $3.500 \mathrm{~m}$ (TEIXEIRA et al., 2003). O Amaranthus cruentus L. foi adaptado ao clima e solo brasileiros pela EmbrapaCerrados (Planaltina-DF) que lançou a variedade BRS - Alegria (SPEHAR et al., 2003).

O grão de $A$. cruentus apresenta cerca de $60 \%$ de amido, $15 \%$ de proteína, $13 \%$ de fibra alimentar, $8 \%$ de lipídeos e $4 \%$ de cinzas (CAPRILES et al., 2008a), o que o destaca dos grãos de cereais (ESCUDERO et al., 2004). O A. cruentus, BRS - Alegria apresenta quantidades consideráveis, expressas em $100 \mathrm{~g}$ a base seca, de fósforo (441 mg), potássio (434 mg), magnésio (254 mg), cálcio (206 mg), sódio (0,6 mg), ferro (12 mg), zinco (5,2 mg), manganês (4 mg), alumínio (4 mg), cobalto (0,06 mg) e selênio (0,02 mg) (MENDONÇA et al., 2005).

$O$ efeito hipocolesterolêmico do grão de amaranto foi associado a compostos presentes em sua composição, tais como o esqualeno, a fibra alimentar, os tocotrienóis, os compostos isoprenoides e a proteína (PLATE e ARÊAS, 2002; MENDONÇA et al., 2009).

Apesar dos benefícios nutricionais e do seu potencial como alimento funcional, o amaranto ainda é um alimento pouco difundido no Brasil. Para o aumento do consumo dessa matéria-prima, preconiza-se a sua incorporação na formulação de produtos e preparações convencionais. Esse tipo de abordagem vem mostrando o potencial do amaranto na panificação, melhorando o valor nutritivo de pães, biscoitos e bolos, gerando produtos aceitos sensorialmente (CAPRILES et al., 2006, 2008b).

Outra aplicação proposta é na elaboração de produtos de conveniência, tais como os snacks extrudados (CHAVEZ-JAUREGUI et al., 2000). Snacks são produtos prontos para o consumo, obtidos por meio da cocção por extrusão de farinhas amiláceas, seguida de secagem e aromatização (MITCHELL e ARÊAS, 1992). Estimativas revelam crescimento anual de cerca de $6 \%$ no mercado mundial de snacks extrudados (PIGNOCCHI, 2007). Dados brasileiros indicam que a produção de snacks praticamente dobrou entre 1990 e 1995 (POLTRONIERI et al., 2000) e que o seu consumo por crianças é semelhante em todas as faixas de renda (AQUINO e PHILIPPI, 2002).

Snacks são elaborados com farinhas amiláceas e aromatizados com uso de lipídeos como agentes fixadores de aroma e sabor. Por essa razão, os snacks extrudados apresentam elevado valor calórico, baixo teor de proteína e de fibra alimentar, e elevadas quantidades de lipídeos (CAPRILES et al., 2007). Várias abordagens vêm sendo testadas para a melhora do valor nutritivo dos snacks extrudados, como a fixação de aroma e sabor no produto por solução contendo fibra solúvel prebiótica, como substituto dos lipídeos (CAPRILES et al., 2009) e a incorporação de matérias-primas com valor nutritivo agregado à formulação.

A utilização do amaranto para elaboração de produtos extrudados resultou em snacks e cereais matinais com melhor valor nutritivo do que os similares comerciais, como observado por Chavez-Jauregui et al. (2000, 2009) e Coelho (2006). A farinha de amaranto desengordurada vem sendo utilizada para elaboração de extrudados expandidos diretos, mas poucos estudos têm explorado a extrusão do grão integral de amaranto (ILO et al., 1999), do fubá de amaranto (DOKIĆ et al., 2009) ou da farinha de amaranto integral (ASCHERI et al., 2005); tal fato se deve, provavelmente, ao alto conteúdo de lipídeos (8\%) do grão de amaranto, que pode atuar como um lubrificante, reduzindo o atrito necessário durante o processo de cocção por extrusão (ILO et al., 1999). A extrusão direta poderia reduzir os custos de produção por meio da eliminação das etapas de moagem e de desengorduramento do grão de amaranto, além de atender a crescente demanda por produtos à base de grãos integrais.

Assim, para verificar a possibilidade da extrusão direta de grão integral de amaranto e suas misturas com 25 e 50\% de fubá de milho proporcionar snacks extrudados com qualidade equivalente àquela obtida por extrusão de farinha de amaranto desengordurada e suas misturas com 25 e 50\% de fubá de milho, foi realizada a pesquisa.

\section{Material e métodos}

Foram utilizadas condições de processamento previamente estabelecidas para a extrusão da farinha de amaranto desengordurada (CHAVEZ-JAUREGUI et al., 
Avaliação da qualidade tecnológica de snacks obtidos por extrusão de grão integral de amaranto ou de farinha de amaranto desengordurada e suas misturas com fubá de milho

CAPRILES, V. D. e AREAS, J. A. G.

2000; COELHO, 2006) e para a aromatização de snacks extrudados por meio de solução contendo fibra solúvel prebiótica, como substituto dos lipídeos (CAPRILES et al., 2009).

\subsection{Material}

Os grãos de Amaranthus cruentus L. BRS-Alegria, cultivados em Brasília-DF, Brasil, foram moídos em moinho de martelos fixos - MML 100 - Astecma, Equipamentos de Moagem Ltda., São Paulo-SP, Brasil - para obtenção da farinha (granulometria $<0,85 \mathrm{~mm}$ ), que foi desengordurada com hexano na proporção de 1:5 (massa:volume).

Para se conseguir o desengorduramento de maiores quantidades de farinha, foi adotado o procedimento padronizado por Mendonça (2006), que constou da utilização de bombonas plásticas com capacidade de $50 \mathrm{~L}$. Para cada $7 \mathrm{~kg}$ de farinha de amaranto, foram utilizados $35 \mathrm{~L}$ de hexano. Essa mistura foi agitada por $4 \mathrm{~h}$ com auxilio de um agitador de tripé, composto por haste com hélice naval em aço inox acoplada a um motor com velocidade de 1730 rpm (Kohlbach Motores Ltda., Jaraguá do Sul-Santa Catarina, Brasil). A mistura foi então decantada e o sobrenadante coletado para posterior recuperação. A farinha foi vertida em papel filtro e o solvente residual evaporado em capela à temperatura ambiente por $24 \mathrm{~h}$. A seguir, a farinha foi seca em estufa com circulação de ar por $24 \mathrm{~h}$, à temperatura de $40^{\circ} \mathrm{C}$, sendo regularmente revolvida para secagem homogênea. A farinha desengordurada seca foi acondicionada em sacos de polietileno, que foram selados e mantidos por uma semana à temperatura ambiente $\left(25^{\circ} \mathrm{C}\right)$ até a sua utilização para produção dos snacks extrudados.

Na etapa de extrusão, foram utilizados grãos integrais de amaranto, farinha de amaranto desengordurada e fubá de milho degerminado (Zea mays L.). Para a aromatização, aroma de bacon (Raí Ingredients Comercial Ltda.), sal micronizado (Norte Salineira S.A. - Indústria e Comércio), água e mistura de inulina e oligofrutose, 1:1 (Beneo ${ }^{\circledR}$ Synergy 1, Orafti N.V., Tienen, Bélgica), de acordo com Capriles et al. (2009).

\subsection{Formulação e processamento dos snacks extrudados}

A Tabela 1 apresenta a composição das matériasprimas utilizadas na elaboração dos snacks extrudados.

Foi utilizado o grão integral de amaranto ou a farinha de amaranto desengordurada isoladamente e em combinação com o fubá de milho nas proporções de 25 e $50 \%$. A farinha de amaranto e as suas combinações tiveram a umidade ajustada para $15 \%$, pois este foi o teor de umidade otimizado para a máxima expansão do extrusado de amaranto (CHAVEZ-JAUREGUI et al., 2000; COELHO, 2006).

Os snacks extrudados foram produzidos de acordo com condições previamente padronizadas (COELHO, 2006; CAPRILES, 2009). Foi utilizada uma extrusora de parafuso único com razão de comprimento/diâmetro (C/D) de 4,6:1 (454:98 mm) e quatro zonas de aquecimento (Inbra 200 - INBRAMAQ, Indústria de Máquinas Ltda, Ribeirão Preto-São Paulo, Brasil). As condições do processo de extrusão foram fixadas em velocidade da rosca de 404 rpm, taxa de compressão da rosca de 1:1, trafila de três furos de $3,17 \mathrm{~mm}$ de diâmetro interno, subtrafila com 154 furos de 3,17 mm de diâmetro interno, "input" de energia de 18 A em 220 V. As zonas de alimentação e de transição (primeira e segunda zonas, respectivamente) foram mantidas em $25^{\circ} \mathrm{C}$, e a terceira zona (zona de alta pressão) e o orifício de saída, em $90^{\circ} \mathrm{C}$.

A aromatização foi realizada de acordo com o processo desenvolvido por Capriles et al. (2009), de forma a substituir a gordura em snacks extrudados e incorporar a mistura de inulina e oligofrutose (1:1), que são fibras solúveis prebióticas. O processo foi padronizado para que o produto final apresentasse, em uma porção (30 g), a quantidade equivalente à ingestão diária de frutanos $(4 \mathrm{~g})$, cujo efeito prebiótico foi observado em seres humanos (RAO, 2001).

Foi utilizada uma solução aromatizante composta por $60 \%$ de sólidos e $40 \%$ de água. Todas as amostras foram aromatizadas com as mesmas quantidades de sal (2\%), aroma (4\%) e mistura de inulina e oligofrutose $(13,3 \%)$. O processo foi realizado em drageador (AIR

Tabela 1. Composição das matérias-primas utilizadas no processo de extrusão.

\begin{tabular}{lccc} 
& Fubá de milho & Grão integral de amaranto & Farinha de amaranto desengordurada \\
Umidade & $12,26 \pm 0,05$ & $9,07 \pm 0,07$ & $11,37 \pm 0,04$ \\
Cinzas & $0,24 \pm 0,05$ & $3,39 \pm 0,01$ & $3,63 \pm 0,00$ \\
Lipídeos & $0,23 \pm 0,03$ & $7,57 \pm 0,10$ & $1,37 \pm 0,04$ \\
Proteína & $7,90 \pm 0,38$ & $16,21 \pm 0,58$ & $17,30 \pm 0,48$ \\
Amido total & $89,39 \pm 0,23$ & $58,00 \pm 0,29$ & $61,89 \pm 0,31$ \\
Fibra alimentar & 2,71 & 14,84 & 15,24 \\
$\quad$ Fibra insolúvel & $2,59 \pm 0,30$ & $12,64 \pm 0,91$ & $13,49 \pm 0,90$ \\
\multicolumn{1}{c}{ Fibra solúvel } & $0,20 \pm 0,04$ & $2,20 \pm 0,34$ & $1,75 \pm 0,10$
\end{tabular}

Resultados das matérias-primas utilizadas na pesquisa, expressos em base seca ( $\mathrm{g} .100 \mathrm{~g}^{-1}$ ). Fonte: Capriles (2009). 
Avaliação da qualidade tecnológica de snacks obtidos por extrusão de grão integral de amaranto ou de farinha de amaranto desengordurada e suas misturas com fubá de milho

CAPRILES, V. D. e AREASS, J. A. G.

400 - Erweka, Heusenstamm, Alemanha) por meio da aspersão da solução aromatizante sobre o snack, seguida de agitação e secagem com ar aquecido a $80{ }^{\circ} \mathrm{C}$ por período de um minuto para cada $100 \mathrm{~g}$ de snack. Esse processo permite que o teor de água adicionado durante a aromatização seja removido e, por isso, este não foi considerado no cálculo da formulação do snack.

Snacks extrudados à base de milho e amaranto na forma de grãos ou de farinha desengordurada (50, 75 e 100\%) foram produzidos sob as mesmas condições de processamento e condimentados no aroma bacon, totalizando seis amostras.

\subsection{Análise dos snacks extrudados}

\subsubsection{Propriedades físicas}

Os snacks extrudados obtidos apresentaram formato cilíndrico e, com o auxílio de um paquímetro, foram medidos o diâmetro e a altura de 30 unidades aleatórias de cada amostra. Esses valores foram utilizados para o cálculo do volume dos snacks segundo a seguinte fórmula: Volume $=\pi r^{2} h$, sendo: $\pi=3,14 ; r^{2}=$ raio ao quadrado; $h$ = altura (CAPRILES et al., 2009). Foram pesadas 30 unidades aleatórias de cada snack em balança analítica e então calculados a densidade pela relação massa/volume (mg. $\mathrm{mm}^{-3}$ ) e o volume específico pela relação volume/massa $\left(\mathrm{mm}^{3} \cdot \mathrm{mg}^{-1}\right.$ ) (ALVAREZMARTINEZ et al., 1988). Também foi determinada a razão de expansão como a razão entre o diâmetro médio do produto extrusado pelo diâmetro do orifício de saída da extrusora.

A força necessária para a quebra do produto foi utilizada para avaliar a crocância, principal propriedade de textura dos snacks extrudados. Esse parâmetro foi avaliado como a força máxima oferecida pela amostra durante o cisalhamento em uma cela do tipo "Warner Bratzler" acoplada ao analisador de textura (TA-XT2i, Stable Micro Systems, Surrey, Reino Unido). A velocidade de corte foi de $1 \mathrm{~mm} \cdot \mathrm{s}^{-1}$. A análise foi realizada em quinze unidades aleatórias de cada amostra. A tensão de cisalhamento dos snacks extrudados foi calculada por meio da divisão da força necessária para a quebra pela área de secção transversal (N.mm²).

O teor de umidade foi determinado por dessecação em estufa a $105^{\circ} \mathrm{C}$, até peso constante, de acordo com o método 950.46 da AOAC (HORWITZ, 2003), e a atividade de água foi determinada em medidor automático (CX-2, Aqualab-Decagon Devices Inc., Washington, Estados Unidos da América), à temperatura ambiente.

A cor das amostras trituradas foi avaliada em triplicata no aparelho Color Quest XE (Hunter Lab, Estados Unidos da América), adotando-se o sistema CIE-Lab, com ângulo de observação de $10^{\circ}$ e iluminante padrão D65, que corresponde à luz natural do dia. Foram medidos os valores de $L^{*}$ ou luminosidade (preto 0/branco 100), a* (verde -/ vermelho +) e b* (azul-/amarelo +).

\subsubsection{Aceitabilidade}

O protocolo experimental foi aprovado pelo Comitê de Ética em Pesquisa da Instituição (protocolo 1404), por atender às exigências de proteção e privacidade dos voluntários, mediante termos de consentimento livre e esclarecido.

Cinquenta e seis provadores não treinados avaliaram a aceitação da cor, da textura e do sabor, e a aceitação global dos snacks em uma escala hedônica estruturada de nove pontos, 1) desgostei muitíssimo; 5) não gostei/ nem desgostei; 9) gostei muitíssimo (STONE e SIDEL, 1985).

Três unidades aleatórias de cada amostra embaladas em sacos de polipropileno codificados com três números aleatórios foram entregues aos provadores, juntamente com a ficha de avaliação e um copo de água para a neutralização entre as amostras. Cada provador avaliou as seis amostras, que foram apresentados de forma monádica e sequencial, utilizando-se blocos completos balanceados para minimizar os efeitos de contraste e de ordem de apresentação das amostras.

\subsection{Análise de dados}

Os resultados foram expressos como média e desvio padrão. Para comparação de médias, os dados foram submetidos à análise de variância (ANOVA) e ao pós-teste de Tukey. Foram calculados os coeficientes de correlação de Pearson e a significância da correlação foi avaliada por meio do teste- $t$ de Student bicaudal. O software MINITAB 15.0 (Minitab Inc., State College, Pensilvânia, Estados Unidos da América) foi utilizado para análise estatística adotando-se nível de significância de $p \leq 0,05$.

\section{Resultados e discussão}

As condições de processamento foram as mesmas para todas as formulações testadas; por isso, as diferenças entre as propriedades dos produtos foram atribuídas à diferença de composição das matériasprimas extrusadas e à interação de seus componentes durante o processo.

\subsection{Expansão e textura dos snacks extrudados}

A expansão dos produtos extrudados é dependente do grau de gelificação do amido e da taxa de evaporação da água no momento da saída do produto da extrusora, que são afetados pelas condições de processo e pela composição da matéria-prima. O amido gelificado contribui com a viscosidade, a elasticidade e a capacidade de 
Avaliação da qualidade tecnológica de snacks obtidos por extrusão de grão integral de amaranto ou de farinha de amaranto desengordurada e suas misturas com fubá de milho

CAPRILES, V. D. e AREASS, J. A. G.

retenção de gás da massa fundida formada durante o processo. Quando o material é forçado pela matriz para sair da extrusora, este expande rapidamente por causa da evaporação instantânea da água, ocasionada pela diferença de pressão entre o interior do equipamento e o ambiente; assim, formam-se bolhas no interior da massa fundida, gerando um produto com diâmetro várias vezes maior que o tamanho do orifício da matriz do equipamento (MITCHELL e ARÊAS, 1992; MERCIER et al., 1998).

É importante monitorar as propriedades de expansão, pois estas estão relacionadas com a textura do produto. Geralmente, a expansão máxima é desejada para snacks extrudados, uma vez que produtos com grande expansão são mais crocantes pelo fato de a estrutura interna apresentar células maiores com paredes finas (MERCIER et al., 1998).

A razão de expansão expressa a expansão radial, ou seja, a expansão que ocorre somente na direção perpendicular ao fluxo da extrusão. A densidade aparente e o volume específico são medidas da expansão volumétrica que é a soma das expansões radial e axial (CHIANG e JOHNSON, 1977; ALVAREZ-MARTINEZ et al., 1988).

A Tabela 2 apresenta a comparação das características de expansão (razão de expansão, densidade e volume específico) e de textura instrumental (força de quebra e tensão de cisalhamento) dos snacks extrudados.

Observa-se, na Tabela 2, que o snack extrudado obtido com $100 \%$ de grão de amaranto se destacou dos demais por apresentar a menor expansão volumétrica (menor razão de expansão, maior densidade e menor volume específico) e, consequentemente, maior força de quebra e tensão de cisalhamento; isso indica que os lipídeos presentes no grão de amaranto $(7,6 \%$, Tabela 1$)$ prejudicaram a expansão e a textura do produto. 0 excesso de lipídeos provoca um efeito lubrificante durante o processo de extrusão, reduzindo a dissipação da energia mecânica e prejudicando o rompimento dos grânulos de amido (ILO et al., 1999). Além disso, as moléculas de lipídeos podem ser incorporadas às hélices de amilose (ARÊAS, 1996). Esses fatores reduzem a quantidade de amido gelificado na massa e, consequentemente, a expansão do extrusado.

Observou-se que quanto maior o teor de grãos de amaranto, menor o volume específico e maior a tensão de cisalhamento dos snacks extrudados $(r=-1,00$; $p=0,011)$. Esse efeito do amaranto integral também foi observado por llo et al. (1999), ao extrusarem misturas de farinha de arroz com 20 a 60\% de grão de amaranto, e por Dokić et al. (2009), ao extrusarem misturas de fubá de milho com 20 e 50\% de fubá de amaranto, e também por Ascheri et al. (2005), ao extrusarem misturas de farinha de arroz com 13 a $47 \%$ de farinha de amaranto integral.

A adição de 25 e $50 \%$ de fubá de milho na formulação dos snacks extrudados obtidos com os grãos de amaranto resultou em produtos significativamente mais expandidos em razão da progressiva redução do teor de lipídeos e do aumento do teor de amido nas formulações. A adição de milho também resultou na redução da força e da tensão de cisalhamento dos produtos. Não foram observadas diferenças de textura instrumental entre os snacks extrudados obtidos com 50 e $75 \%$ de grão de amaranto.

Ao se comparar os snacks extrudados obtidos com farinha de amaranto desengordurada, constatou-se que apenas a razão de expansão sofreu aumento significativo e a partir de $50 \%$ de adição de fubá de milho. Não foi observado efeito da adição de milho na densidade, no volume específico e nem na textura instrumental dos snacks obtidos com farinha desengordurada; possivelmente, porque foi garantida a presença da quantidade mínima de amido necessário para uma boa expansão do produto.

Apenas os resultados de razão de expansão e de densidade dos snacks extrudados obtidos com a combinação de farinha de amaranto desengordurada e fubá de milho (Tabela 2) foram comparáveis aos obtidos por Chavez-Jauregui et al. (2000), que otimizaram o processo de extrusão para obtenção de snacks à base

Tabela 2. Expansão e textura dos snacks extrudados obtidos a partir do grão ou da farinha de amaranto desengordurada e da combinação destes com fubá de milho.

\begin{tabular}{|c|c|c|c|c|c|c|}
\hline & \multicolumn{3}{|c|}{ Proporção de grão na formulação } & \multicolumn{3}{|c|}{$\begin{array}{l}\text { Proporção de farinha desengordurada } \\
\text { na formulação }\end{array}$} \\
\hline & $50 \%$ & $75 \%$ & $100 \%$ & $50 \%$ & $75 \%$ & $100 \%$ \\
\hline Razão de e & $3,17^{a} \pm 0,14$ & $2,87^{b} \pm 0,26$ & $2,08^{d} \pm 0,16$ & $2,96^{\mathrm{ab}} \pm 0,16$ & $2,50^{c} \pm 0,32$ & $2,62^{c} \pm 0,15$ \\
\hline Densidade (mg. $\left.\mathrm{mm}^{-3}\right)$ & $0,25^{b} \pm 0,06$ & $0,27^{b} \pm 0,07$ & $0,65^{a} \pm 0,02$ & $0,22^{b} \pm 0,06$ & $0,24^{b} \pm 0,08$ & 0,04 \\
\hline Volume específico $\left(\mathrm{mm}^{3} \cdot \mathrm{mg}^{-1}\right)$ & $4,26^{b} \pm 1,08$ & $3,93^{b} \pm 1,07$ & $1,76^{c} \pm 0,78$ & $4,86^{\mathrm{ab}} \pm 1,38$ & $4,67^{\mathrm{ab}} \pm 1,53$ & $5,66^{a} \pm 1,21$ \\
\hline Força de quebra (N) & $43,16^{b} \pm 7,93$ & $46,80^{\mathrm{ab}} \pm 7,55$ & $52,60^{a} \pm 9,6$ & $29,94^{c} \pm 4,68$ & $28,59^{c} \pm 4,04$ & $25,51^{c} \pm 4,22$ \\
\hline Tensão de cisalhamer & $0,18^{b} \pm 0,03$ & $0,21^{b} \pm 0,05$ & $0,38^{a} \pm 0,10$ & $0,11^{c} \pm 0,02$ & $0,12^{c} \pm 0,02$ & $0,11^{c} \pm 0,01$ \\
\hline Umidade (\%) & $7,82^{b} \pm 0,35$ & $8,21^{\mathrm{ab}} \pm 0,30$ & $8,09^{a b} \pm 0,09$ & $8,91^{a} \pm 0,0$ & $8,82^{\mathrm{a}} \pm 0,11$ & $8,91^{a} \pm 0,47$ \\
\hline Atividade de água & $0,45^{b c} \pm 0,00$ & $0,47^{a} \pm 0,00$ & $0,47^{a} \pm 0,00$ & $0,45^{b} \pm 0,00$ & $0,48^{a} \pm 0,00$ & $0,44^{\circ} \pm 0,00$ \\
\hline
\end{tabular}

As médias seguidas por letras diferentes, na mesma linha, diferem entre si $(p \leq 0,05)$. 
Avaliação da qualidade tecnológica de snacks obtidos por extrusão de grão integral de amaranto ou de farinha de amaranto desengordurada e suas misturas com fubá de milho

CAPRILES, V. D. e AREAS, J. A. G.

de farinha de amaranto desengordurada e obtiveram, nas melhores condições de processamento, razão de expansão de 2,65 a 2,84, força de quebra de 13,8 a $14,5 \mathrm{~N}$, tensão de cisalhamento de 0,281 a 0,325 N.mm² e densidade de 0,22 a 0,24 mg. $\mathrm{mm}^{-3}$.

Os snacks apresentam baixos valores de umidade, inferiores a 9\%, e de atividade de água, inferiores a 0,5 , sendo considerados estáveis microbiologicamente (ASCHERI et al., 2005).

\subsection{Cor dos snacks extrudados}

A adição de milho à formulação fez com que os snacks ficassem mais claros (aumento do valor de $L^{*}$ ) e amarelados (aumento dos valores da coordenada $b^{\star}$ ), como pode ser observado na Tabela 3. Não foram observados efeitos na coordenada cromática $a^{*}$. As amostras obtidas com as mesmas proporções de matériaprima apresentaram valores próximos para os parâmetros de cor, indicando que o estado físico do amaranto e o teor de lipídeos não exerceram grande influência na cor dos snacks.

\subsection{Aceitabilidade dos snacks extrudados}

A aceitação é um atributo crítico, porque é uma das propriedades que atraem os consumidores de snacks. Os vários parâmetros de aceitação dos snacks extrudados são a aparência, a cor, a textura, o sabor e o odor. A aceitação da textura é o atributo mais importante nesses produtos (HEIDENREICH et al., 2004; MAZUMDER et al., 2007). A Tabela 4 apresenta a comparação da aceitabilidade sensorial dos snacks.

Observou-se que quanto menor o teor de amaranto na formulação dos snacks extrudados obtidos com os grãos ou com a farinha de amaranto desengordurada, maior a saturação da cor amarela (maiores os valores de $\left.b^{*}\right)$ e, consequentemente, maior a aceitação da cor do produto $(r=1,00 ; p<0,02)$. A adição de $50 \%$ de fubá de milho resultou em aumento significativo da aceitação da cor, possivelmente porque a cor das amostras ficou mais próxima da cor de um snack convencional, obtido apenas com fubá de milho e corante, geralmente o urucum. Não houve diferença significativa de aceitação da cor entre os produtos obtidos com as mesmas proporções de amaranto e milho em função do estado físico do amaranto.

A amostra obtida apenas com grãos de amaranto apresentou aceitação da textura significativamente menor que a das demais amostras, em função de sua maior tensão de cisalhamento, que é uma consequência de sua estrutura mais densa. Apesar de detectadas instrumentalmente, não foram observadas diferenças significativas de aceitação da textura entre as demais formulações. Alguns provadores comentaram que o snack extrudado obtido com $100 \%$ de farinha de amaranto desengordurada aderia ao dente e que esta característica era desagradável.

De forma geral, observou-se que as amostras apresentaram baixa aceitação do sabor, mostrando que nem a adição de milho nas concentrações testadas e nem o aroma de bacon foram suficientes para amenizar o gosto residual do amaranto. Os snacks obtidos com grãos de amaranto apresentaram aceitação do sabor significativamente menor que a observada para as formulações obtidas com farinha desengordurada. Acredita-se que o maior teor de lipídeos presente no snack elaborado com os grãos tenha intensificado a percepção do gosto residual do amaranto.

Mesmo com a adição de fubá de milho, os snacks à base de grão de amaranto foram rejeitados (aceitação global entre 3 e 4, indicando 'desgostei regularmente'

Tabela 3. Parâmetros de cor dos snacks extrudados obtidos a partir do grão ou da farinha de amaranto desengordurada e da combinação destes com fubá de milho.

\begin{tabular}{|c|c|c|c|c|c|c|}
\hline & \multicolumn{3}{|c|}{ Proporção de grão na formulação } & \multicolumn{3}{|c|}{ Proporção de farinha desengordurada na formulação } \\
\hline & $50 \%$ & $75 \%$ & $100 \%$ & $50 \%$ & $75 \%$ & $100 \%$ \\
\hline$L^{*}$ & $65,95^{a} \pm 0,04$ & $62,02^{c} \pm 0,77$ & $59,99^{d} \pm 0,32$ & $63,71^{b} \pm 0,05$ & $60,38^{d} \pm 0,60$ & $60,67^{d} \pm 0,38$ \\
\hline$a^{*}$ & $6,18^{d} \pm 0,11$ & $6,52^{c} \pm 0,14$ & $6,17^{d} \pm 0,08$ & $7,41^{a} \pm 0,10$ & $7,23^{\mathrm{ab}} \pm 0,12$ & $6,93^{b} \pm 0,07$ \\
\hline$b^{*}$ & $22,48^{a} \pm 0,21$ & $19,69^{b} \pm 0,11$ & $17,46^{c} \pm 0,29$ & $22,90^{\mathrm{a}} \pm 0,17$ & $19,62^{b} \pm 0,09$ & $17,98^{c} \pm 0,40$ \\
\hline
\end{tabular}

As médias seguidas por letras diferentes, na mesma linha, diferem entre si $(p \leq 0,05)$.

Tabela 4. Aceitação dos snacks extrudados obtidos a partir do grão ou da farinha de amaranto desengordurada e da combinação destes com fubá de milho.

\begin{tabular}{|c|c|c|c|c|c|c|}
\hline \multirow[t]{2}{*}{ Parâmetros } & \multicolumn{3}{|c|}{ Proporção de grão na formulação } & \multicolumn{3}{|c|}{ Proporção de farinha desengordurada na formulação } \\
\hline & $50 \%$ & $75 \%$ & $100 \%$ & $50 \%$ & $75 \%$ & $100 \%$ \\
\hline Cor & $6,2^{\mathrm{a}} \pm 1,4$ & $5,1^{b} \pm 1,6$ & $4,2^{\circ} \pm 1,7$ & $6,1^{\mathrm{a}} \pm 1,5$ & $5,4^{\mathrm{ab}} \pm 1,5$ & $5,0^{\mathrm{bc}} \pm 1,6$ \\
\hline Textura & $6,0^{\mathrm{a}} \pm 1,6$ & $6,1^{a} \pm 2,0$ & $4,7^{b} \pm 1,9$ & $6,5^{a} \pm 1,3$ & $6,8^{\mathrm{a}} \pm 1,3$ & $6,5^{\mathrm{a}} \pm 1,6$ \\
\hline Sabor & $4,4^{\mathrm{bc}} \pm 2,1$ & $4,2^{\mathrm{c}} \pm 2,0$ & $3,4^{c} \pm 1,9$ & $5,8^{\mathrm{a}} \pm 1,6$ & $5,3^{\mathrm{ab}} \pm 1,8$ & $5,3^{a b} \pm 1,9$ \\
\hline Global & $4,6^{b} \pm 2,0$ & $4,4^{b c} \pm 2,0$ & $3,4^{\mathrm{c}} \pm 1,7$ & $5,7^{a} \pm 1,5$ & $5,2^{\mathrm{ab}} \pm 1,8$ & $5,2^{\mathrm{ab}} \pm 1,8$ \\
\hline
\end{tabular}

As médias seguidas por letras diferentes, na mesma linha, diferem entre si $(p \leq 0,05)$. 
Avaliação da qualidade tecnológica de snacks obtidos por extrusão de grão integral de amaranto ou de farinha de amaranto desengordurada e suas misturas com fubá de milho

CAPRILES, V. D. e AREAS, J. A. G.

e 'desgostei ligeiramente'), pois apresentaram baixa expansão, textura dura, cor escura e forte sabor residual. Já os snacks extrudados obtidos com farinha de amaranto desengordurada isoladamente e em combinação com fubá de milho apresentaram maior expansão, textura crocante e cor mais clara; e, por isso, maior aceitabilidade (aceitação global $\geq 5$ ).

O grau de aceitabilidade dos snacks extrudados à base de farinha de amaranto desengordurada desenvolvidos é próximo aquele observado por ChavezJauregui et al. (2009) para snacks extrudados à base de amaranto, que proporcionou aceitação de 6,3 $\pm 2,1$, e da mistura de $20 \%$ de milho com $80 \%$ de amaranto, que proporcionou aceitação de 6,0 $\pm 1,8$, tendo sido estes também condimentados com o aroma bacon. Deve-se considerar que os snacks desenvolvidos por ChavezJauregui et al. (2009) foram aromatizados com 22\% de gordura vegetal hidrogenada como agente fixador do aroma. Capriles et al. $(2007,2009)$ observaram que snacks extrudados de milho aromatizados com essa quantidade de gordura apresentam aceitação significativamente maior do que os snacks de milho aromatizados com a solução aromatizante contendo frutanos $(7,50 \pm 1,20$ versus $6,6 \pm 1,7)$.

Os novos snacks extrudados de amaranto obtidos com substituição da gordura por fibras prebióticas apresentam dupla vantagem - redução de lipídeos e aumento do teor de fibra alimentar - e obtiveram o mesmo grau de aceitação observado anteriormente.

Embora os consumidores desejem a inclusão de grãos inteiros (EASTAMAN e LEE, 2005) por causa da sua vantajosa composição nutricional, os resultados mostram que a extrusão de formulações com 50, 75 e $100 \%$ de grão de amaranto resulta em produtos rejeitados sensorialmente.

Observa-se a necessidade de se melhorarem a cor e o sabor dos snacks extrudados à base de farinha de amaranto desengordurada, o que pode ser obtido por meio de mudanças nas condições de processamento, como, por exemplo, a configuração da extrusora (CAPRILES e AREAAS, 2011).

\section{Conclusões}

A extrusão de grãos de amaranto gerou snacks extrudados rejeitados sensorialmente por apresentarem coloração escura, baixa expansão, elevada tensão de cisalhamento e forte sabor residual. A adição de 25 e $50 \%$ de fubá de milho nessa formulação ocasionou clareamento, aumento da expansão e diminuição da tensão de cisalhamento, mas não foi suficiente para elevar a aceitação dos produtos.

Por outro lado, a produção de snacks extrudados à base de farinha de amaranto desengordurada proporciona produtos com melhor aceitação. A adição de $50 \%$ de fubá de milho na formulação resulta em aumento da aceitabilidade sensorial do produto; mesmo assim, os resultados mostram a necessidade de melhoria nas características de cor e de sabor.

\section{Agradecimentos}

Aos voluntários que participaram da análise sensorial. Às indústrias que cederam amostras de seus ingredientes para a realização deste trabalho. À Fundação de Amparo à Pesquisa do Estado de São Paulo (FAPESP) pela concessão da Bolsa de Doutorado Direto (Processo $n^{\circ}$ 04/14127-3).

\section{Referências}

ALVAREZ-MARTINEZ, L.; KONDURY, K.; HARPER, J. A. A general-mode for expansion of extruded products. Journal of Food Science, Chicago, v. 53, n. 2, p. 609-615, 1988. http:// dx.doi.org/10.1111/j.1365-2621.1988.tb07768.x

AQUINO, R.C.; PHILIPPI, S.T. Consumo infantil de alimentos industrializados e renda familiar na cidade de São Paulo. Revista de Saúde Pública, São Paulo, v. 36, n. 6, p. 655-660, 2002. http://dx.doi.org/10.1590/S0034-89102002000700001

ARÊAS, J. A. G. Interações moleculares do amido durante o processo de extrusão. Boletim da Sociedade Brasileira de Ciência e Tecnologia de Alimentos - SBCTA, Campinas, v. 30, n. 1, p. 28-30, 1996.

ASCHERI, J. L. R.; RIBEIRO, M. M.; CARVALHO, C. P.; ASCHERI, D. P. R. Isotermas de adsorcion de agua y estabilidad de harinas extruídas de amaranto, arroz y maíz: estudio comparativo. Alimentaria, Madrid, v. 42, n. 363, p. 100-107, 2005.

CAPRILES, V. D.; ARÊAS, J. A. G. Redução da razão comprimento/diâmetro da extrusora e aumento da aceitabilidade de snacks à base de amaranto. Brazilian Journal of Food Technology, Campinas, v. 14, n. 1, p. 19-26, 2011. http://dx.doi. org/10.4260/BJFT2011140100003

CAPRILES, V. D.; COELHO, K. D.; GUERRA-MATIAS, A. C.; ARÊAS, J. A. G. Efeito da adição de amaranto na composição e na aceitabilidade do biscoito tipo cookie e do pão de forma.

Alimentos e Nutrição, Araraquara, v. 17, n. 3, p. 269-274, 2006.

CAPRILES, V.D.; SOARES, R.A.M.; ARÊAS, J.A.G. Development and assessment of acceptability and nutritional properties of a light snack. Ciência e Tecnologia de Alimentos, Campinas, v. 27 , n. 3, p. 562-566, 2007. http://dx.doi.org/10.1590/S010120612007000300020

CAPRILES, V. D.; COELHO, K. D.; GUERRA-MATIAS, A. C.; AREAAS, J. A. G. Effects of processing methods on amaranth starch digestibility and predicted glycemic index. Journal of Food Science, Chicago, v. 73, n. 7, p. H160-H164, 2008a. http:// dx.doi.org/10.1111/j.1750-3841.2008.00869.x 
Avaliação da qualidade tecnológica de snacks obtidos por extrusão de grão integral de amaranto ou de farinha de amaranto desengordurada e suas misturas com fubá de milho

CAPRILES, V. D. e AREAS, J. A. G.

CAPRILES, V. D.; ALMEIDA, E. L.; FERREIRA, R. E.; ARÊAS, J. A. G.; STEEL, C. J.; CHANG, Y. K. Physical and sensory properties of regular and reduced-fat pound cakes with added amaranth flour. Cereal Chemistry, Minnesota, v. 85, n. 5, p. 614-618, 2008b. http://dx.doi.org/10.1094/CCHEM-85-5-0614

CAPRILES, V. D.; SOARES, R. A. M.; PINTO e SILVA, M. E. M.; ARÊAS, J. A. G. Effect of fructans-based fat replacer on chemical composition, starch digestibility and sensory acceptability of corn snacks. International Journal of Food Science \& Technology, Malden, v. 44, n. 10, p. 1895-1901, 2009. http:// dx.doi.org/10.1111/j.1365-2621.2009.01915.x

CAPRILES, V. D. Otimização de Propriedades Nutricionais e Sensoriais de Produtos à Base de Amaranto Enriquecidos com Frutanos, para Intervenção em Celíacos. 2009. 198 f. Tese (Doutorado em Ciências)-Faculdade de Saúde Pública , Universidade de São Paulo, São Paulo, 2009.

CHAVEZ-JAUREGUI, R.; PINTO e SILVA, M. E. M.; ARÊAS, J. A. G. Extrusion cooking process for amaranth (Amaranthus caudatus L.). Journal of Food Science, Chicago, v. 65, n. 6, p. 1009-1015, 2000. http://dx.doi.org/10.1111/j.1365-2621.2000. tb09408.x

CHAVEZ-JAUREGUI, R.; PINTO e SILVA, M. E. M.; ARÊAS, J. A. G. Storage effect on the acceptability of snacks made of pure amaranth and blends of amaranth and corn and chickpea. Alimentaria, Madrid, v. 405, p. 117-121, 2009

CHIANG, B.; JOHNSON, J. Gelatinization of starch in extruded products. Cereal Chemistry, Minnesota, v. 54, n. 3, p. 436-443, 1977.

COELHO, K. D. Desenvolvimento e Avaliação da Aceitação de Cereais Matinais e Barras de Cereais à Base de Amaranto (Amaranthus cruentus L). 2006. 89 f. Dissertação (Mestrado em Nutrição Humana Aplicada)- Universidade de São Paulo, São Paulo, 2006.

DOKIĆ, L. P.; BODROŽA-SOLAROV, M. I.; HADNAĐEV, M. S.; NIKOLIĆ, I. R. Properties of extruded snacks supplemented with amaranth grain grits. Acta Periodica Technologica, Novi Sad, v. 40 , p. $17-24,2009$

EASTAMAN, J.; LEE, G. Whole grains in extruded products. Cereal Foods World, Minnesota, v. 50, n. 4, p. 168-172, 2005.

ESCUDERO, N.; DE ARELLANO, M.; LUCO, J.; GIMENEZ, M.; MUCCIARELLI, S. Comparison of the chemical composition and nutritional value of Amaranthus cruentus flour and its protein concentrate. Plant Foods for Human Nutrition, Netherlands, v. 59, n. 1, p. 15-21, 2004. http://dx.doi.org/10.1007/s11130004-0033-3

FERREIRA, T. A. P. C.; GUERRA-MATIAS, A. C.; ARÊAS, J. A. G. Características nutricionais e funcionais do Amaranto (Amaranthus sp.). Nutrire, São Paulo, v. 32, n. 2, p. 91-116, 2007 HEIDENREICH, S.; JAROS, D.; ROHM, R.; ZIENS, A. Relationship between water activity and crispness of extruded rice crisps.
Journal of Texture Studies, Trumbull, v. 35, n. 6, p. 621-633, 2004. http://dx.doi.org/10.1111/j.1745-4603.2004.35513.x

HORWITZ, W. (Ed.). Official Methods of Analysis of the Association of Official Analytical Chemists. 17. ed. 2. rev. Gaithersburg: AOAC, 2003.

ILO, S.; LIU, Y.; BERGHOFER, E. Extrusion cooking of rice flour and amaranth blends. Food Science and TechnologyLebensmittel Wissenschaft \& Technologie, Oxford, v. 32, n. 2, p. 79-88, 1999. http://dx.doi.org/10.1006/fstl.1998.0497

MAZUMDER, P.; ROOPA, B. S.; BHATTACHARYA, S. Textural attributes of a model snack food at different moisture contents. Journal of Food Engineering, Oxford, v. 79, n. 2, p. 511-516, 2007. http://dx.doi.org/10.1016/j.jfoodeng.2006.02.011

MENDOÇA, X. M. F. D.; ASCHERI, J. L.R.; ASCHERI, D. P. R.; MAIA, M. C. A. Extrusión de harina mixta de amaranto integral y arroz: Parte 1. Caracterización físicoquímica. Alimentaria, Madrid, v. 42, n. 367, p. 74-83, 2005.

MENDONÇA, S. Efeito Hipocolesterolemizante da Proteína de Amaranto (Amranthus cruentus BRS-Alegria) em Hamsters. 2006. 206 f.Tese (Doutorado em Saúde Pública)-Faculdade de Saúde Pública, Universidade de São Paulo, São Paulo, 2006.

MENDONÇA, S.; SALDIVA, P.; CRUZ, R.; ARÊAS, J.A.G. Amaranth protein presents cholesterol-lowering effect. Food Chemistry, Oxford, v. 116, n. 3, p. 738-742, 2009. http://dx.doi. org/10.1016/j.foodchem.2009.03.021

MERCIER, C.; LINKO, P.; HARPER, J. M. Extrusion Cooking. 2. ed. Saint Paul: American Association of Cereal Chemists, 1998. $471 \mathrm{p}$.

MITCHELL, J. R.; AREAAS, J. A. G. Strutural in biopolymers during extrusion. In: KOKINI, J. L.; HO, C. T.; KARWE, M. V. (Eds.). Food extrusion Science and Technology. New York: Marcel Dekker, 1992.

PIGNOCCHI, G. O mercado mundial e brasileiro de snacks: presente e futuro. In: SEMINÁRIO VIABILIZANDO A INOVAÇÃO DE CHOCOLATES, CONFEITOS, PRODUTOS DE PANIFICAÇÃO E CEREAIS COMO SNACKSDE CONVENIÊNCIA E SAÚDE, 2007, Campinas. Anais... Instituto de Tecnologia de Alimentos - ITAL, 2007. CD-ROM.

PLATE, A.; ARÊAS, J. A. G. Cholesterol-lowering effect of extruded amaranth (Amaranthus caudatus L.) in hypercholesterolemic rabbits. Food Chemistry, Oxford, v. 76, n. 1, p. 1-6, 2002. http:// dx.doi.org/10.1016/S0308-8146(01)00238-2

POLTRONIERI, F.; ARÊAS, J. A. G.; COLLI, C. Extrusion and iron bioavaibility in chickpea (Cicer arietinum L,). Food Chemistry, Oxford, v. 70, n. 2, p. 175-180, 2000. http://dx.doi.org/10.1016/ S0956-7135(99)00113-9

RAO, $V$. The prebiotic properties of oligofructose at low intake levels. Nutrition Research, Oxford, v. 21, n. 6, p. 843-848, 2001. http://dx.doi.org/10.1016/S0271-5317(01)00284-6 
Avaliação da qualidade tecnológica de snacks obtidos por extrusão de grão integral de amaranto ou de farinha de amaranto desengordurada e suas misturas com fubá de milho

CAPRILES, V. D. e AREAS, J. A. G.

SPEHAR, C.; TEIXEIRA, D. L.; CABEZAS, W. A. R. L.; ERASMO, E. A. L. Amaranth BRS Alegria: alternative for diversification of cropping systems. Pesquisa Agropecuária Brasileira, Brasília, v. 38, n. 5, p. 659-663, 2003 http://dx.doi.org/10.1590/S0100204X2003000500015

STONE, H.; SIDEL, J. L. Sensory Evaluation Practices. London: Academic Press, 1985.
TEIXEIRA, D. L.; SPEHAR, C. R.; SOUZA, L. A. C. Caracterização agronômica de amaranto para cultivo na entressafra no Cerrado. Pesquisa Agropecuária Brasileira, Brasília, v. 38, n. 1, p. 45-51, 2003. http://dx.doi.org/10.1590/S0100-204X2003000100006

TEUTONICO, R.; KNORR, D. Amaranth: Composition, properties and applications of a rediscovered food crop. Food Technology, Chicago, v. 39, n. 4, p. 49-59, 1985 